Pacific Journal of Mathematic 


\title{
NOETHER'S THEOREM FOR PLANE DOMAINS WITH HYPERELLIPTIC DOUBLE
}

\author{
WILliAM H. BARKER II
}

This paper is motivated by the observation that Noether's theorem for quadratic differentials fails for hyperelliptic Riemann surfaces. In this paper we provide an appropriate substitute for Noether's theorem which is valid for plane domains with hyperelliptic double. Our result is somewhat more explicit than Noether's, and, in contrast with the case of nonhyperelliptic surfaces, it provides a basis for the (even) quadratic differentials which holds globally for all domains with hyperelliptic double. An important fact which plays a significant role in these considerations is that no two normal differentials of the first kind can have a common zero on a domain with hyperelliptic double.

Let $W$ be a closed Riemann surface of genus $g \geqq 1$. We wish to consider the class of analytic quadratic differentials on $W$. In terms of a local parameter $z=z(p), p \in W$, recall that an analytic quadratic differential has the representation $f(z) d z^{2}$, where $f(z)$ is a regular analytic function of the variable $z$. The analytic quadratic differentials form a complex linear space of dimension $3 g-3$ for $g \geqq 1$ and of dimension 1 for $g=1$. Additionally, the product of two Abelian differentials of the first kind is an analytic quadratic differential. Noether's theorem is fundamental in that it provides for a basis for the analytic quadratic differentials on a nonhyperelliptic Riemann surface in terms of products of Abelian differentials of the first kind. See, for example, Hensel and Landsberg [4], p. 502.

In contrast, it is a direct computation that on a hyperelliptic Riemann surface of genus $g \geqq 1$, the products $\theta_{i} \theta_{l}$ of Abelian differentials of the first kind span a complex linear space of dimension $2 g-1$. Thus, in particular, Noether's theorem fails in the case of hyperelliptic surfaces of genus $g \geqq 3$. In this paper we obtain an appropriate substitute for Noether's thoerem in the case of hyperelliptic surfaces obtained as doubles of plane domains.

Definition 1. For each $n \geqq 1$ let $\mathscr{A}_{n}$ be the class of all domains whose boundary is formed by $n$ disjoint piecewise analytic curves. We shall denote by $H_{n}$ the class of all domains in $\mathscr{A}_{n}$ which possess a hyperelliptic double, and by $\Sigma_{n}$ the class of all domains which are the exterior of a system of $n$ slits taken from the real axis. 
We established in [1] that each domain in $H_{n}$ can be mapped analytically and univalently onto a domain in $\Sigma_{n}$.

Suppose now that $D \in \Sigma_{n}$, and that the point at infinity is in $D$. The boundary of $D$ is then formed by the $n$ disjoint segments of the real axis

$$
\gamma_{\jmath}=\left[r_{2 j+1}, r_{2 \jmath+2}\right], \quad j=0,1,2, \cdots, n-1
$$

and we shall find it convenient to order the $\gamma_{J}$ with the assumption

$$
r_{1}<r_{2}<\cdots<r_{2 n}
$$

We also have the complementary segments

$$
\begin{gathered}
\alpha_{j}=\left[r_{2 \jmath+2}, r_{2 \jmath+3}\right], \quad j=0,1,2, \cdots, n-2, \\
\text { and } \alpha_{n-1}=\left[r_{2 n}, \infty\right] \cup\left[-\infty, r_{1}\right] .
\end{gathered}
$$

The structure polynomial $q(z)$ for $D$ is defined by $q(z)=$ $\Pi_{j=1}^{2 n}\left(z-r_{\jmath}\right)$. It is easy to see that the double of $D$ is the hyperelliptic Riemann surface given by $w^{2}=q(z)$.

Let $\omega_{\mu}$ be the harmonic measure in $D$ with boundary values $\delta_{\mu \nu}$ on $\gamma_{\nu}$. Each $\omega_{\mu}$ is the real part of a multivalued analytic function $w_{\mu}(z)=$ $\omega_{\mu}(z)+i \sigma_{\mu}(z)$ whose derivative is single valued on $D$. We define the induction coefficients $P_{\mu \nu}, \mu, \nu=0,1,2, \cdots, n-1$, by

$$
P_{\mu \nu}=-\frac{1}{2 \pi} \int_{\gamma \nu} \frac{\partial \omega_{\mu}}{\partial n} d s=\frac{1}{2 \pi i} \int_{\gamma \nu} w_{\mu}^{\prime}(z) d z
$$

Observe that the study of the Abelian differentials of the first kind on the double of $D$ is equivalent to the study of the differentials $w_{\mu}^{\prime}(z) d z$ on $D$.

THEOREM 1. If $D \in \Sigma_{n}$, and $\gamma_{\mu}$ is a boundary segment of $D$, then $w_{\mu}^{\prime}(z)=\overline{w_{\mu}^{\prime}(\bar{z})}$, and the zeros of $w_{\mu}^{\prime}(z)$ are all simple and are located, one each, on the $n-2$ open segments of the extended real axis complementary to the boundary of $D$ and not adjacent to $\gamma_{\mu}$.

Proof. To simplify our notation, we will prove Theorem 1 only for the differential $w_{0}^{\prime}(z)$. From the definition of $w_{0}(z)$ we have

$$
w_{0}^{\prime}(z)=\frac{\partial}{\partial x} \omega_{0}(z)-i \frac{\partial}{\partial y} \omega_{0}(z)
$$

But observe that $\omega_{0}(z)=\omega_{0}(\bar{z})$, whence for $z=x$ real in $D$, 


$$
\frac{\partial}{\partial y} \omega_{0}(x)=0
$$

and so in this case equation (4) becomes

$$
w_{0}^{\prime}(x)=\frac{\partial}{\partial x} \omega_{0}(x), x \text { real, } x \in D
$$

We have also $w_{0}^{\prime}(z)=\overline{w_{0}^{\prime}(\bar{z})}$.

Now restrict $\omega_{0}$ to one of the intervals $\alpha_{1}, \alpha_{2}, \cdots, \alpha_{n-2}$, say to $\alpha_{\zeta}$. From the boundary behavior of $\omega_{0}$, we see that $\omega_{0}$ is zero at both endpoints of $\alpha_{\zeta}$ and that $\omega_{0}(x)>0$ for $x$ in the interior of $\alpha_{\zeta}$. It follows that $\omega_{0}$ achieves a maximum at some interior point $x_{\zeta}$ of $\alpha_{\zeta}$, and at this point we have $(\partial / \partial x) \omega_{0}\left(x_{\zeta}\right)=0$ and so by equation $(6), w_{0}^{\prime}\left(x_{\zeta}\right)=0$.

Finally, it is known that $w_{0}^{\prime}(z) d z$ can possess at most $n-2$ zeros in the interior of $D$, and thus Theorem 1 follows.

Corollary 1. For a domain in the class $\Sigma_{n}$, if $w_{\mu}^{\prime}\left(z_{0}\right)=0$, then $w_{\mu}^{\prime \prime}\left(z_{0}\right)<0$.

Proof. For $z$ real, $w_{\mu}^{\prime \prime}(z)=\left(\partial^{2} / \partial x^{2}\right) \omega_{\mu}(z)$. Moreover, the condition $w_{\mu}^{\prime}\left(z_{0}\right)=0$ implies that $z_{0}$ is real and that $\omega_{\mu}(z)$ has a local maximum at $z_{0}$; thus $w_{\mu}^{\prime \prime}\left(z_{0}\right) \leqq 0$. But all of the zeros of $w_{\mu}^{\prime}(z)$ are simple and the result follows.

The following result is an immediate consequence of Theorem 1 and the representation of Abelian differentials of the first kind on hyperelliptic surfaces. See, for example, Springer [6], §10-10.

THEOREM 2. For a domain in the class $\Sigma_{n}$ whose structure polynomial is $q(z)$, we have

$$
w_{\mu}^{\prime}(z) d z=\frac{p_{\mu}(z)}{\sqrt{q(z)}} d z
$$

where $p_{\mu}(z)$ is a real polynomial of degree at most $n-2$, all of whose roots are real.

Corollary 2. If $D \in \Sigma_{n}$ has structure polynomial $q(z)$, and if $p(z)$ is a polynomial with real coefficients of degree at most $n-2$, then $p(z) / \sqrt{q(z)}$ can be expressed as a real linear combination of $w_{1}^{\prime}(z), w_{2}^{\prime}(z), \cdots, w_{n-1}^{\prime}(z)$.

Proof. The differentials $w_{1}^{\prime}(z), \cdots, w_{n-1}^{\prime}(z)$ are linearly independent, and, therefore, so also are the polynomials $p_{1}(z), \cdots, p_{n-1}(z)$. 
Our concern now is with products of the form $w_{\mu}^{\prime}(z) w_{\nu}^{\prime}(z) d z^{2}$. Observe that if $D$ is a domain with analytic boundary, then $w_{\mu}^{\prime}(z) w_{\nu}^{\prime}(z) d z^{2}$ is negative and so, in particular, real. It is to preserve this property that we shall consider real rather than complex vector spaces.

Definition 2. For $D \in H_{n}$, we shall denote by $\Gamma^{2}(D)$ the real linear space generated by the products $w_{\mu}^{\prime}(z) w_{\nu}^{\prime}(z) d z^{2}, \mu, \nu=$ $1,2, \cdots, n-1$.

If $D \in \Sigma_{n}, n=2,3, \cdots$, with structure polynomial $q(z)$, it is an immediate consequence of Theorem 2 that

$$
\Gamma^{2}(D)=\left\{\frac{p(z)}{q(z)} d z^{2}: p(z)\right. \text { is a polynomial with real coefficients of }
$$

$$
\text { degree at most } 2 n-4\} \text {. }
$$

In particular, we see that if $D \in H_{n}$, then $\Gamma^{2}(D)$ is a real linear space of real dimension $2 n-3, n=2,3, \cdots$.

LemMA 1. If $D$ is a domain in the class $\Sigma_{n}$, and if, for some enumeration of the boundary segments of $D$, the differentials $w_{1}^{\prime}(z) d z$ and $w_{2}^{\prime}(z) d z$ have no common zeros, then

$$
\begin{array}{r}
w_{1}^{\prime}(z)^{2} d z^{2}, w_{1}^{\prime}(z) w_{2}^{\prime}(z) d z^{2}, \cdots, w_{1}^{\prime}(z) w_{n-1}^{\prime}(z) d z^{2}, \\
w_{2}^{\prime}(z)^{2} d z^{2}, \cdots, w_{2}^{\prime}(z) w_{n-1}^{\prime}(z) d z^{2}
\end{array}
$$

are linearly independent and so form a basis for $\Gamma^{2}(D)$.

Proof. Let $D$ be a domain in the class $\Sigma_{n}$ with structure polynomial $q(z)$. Each of the differentials $w_{\mu}^{\prime}(z) d z$ is then of the form $p_{\mu}(z) / \sqrt{q(z)} d z$ where $p_{\mu}(z)$ is a polynomial of degree at most $n-$ 2. Since the linear dependence properties of the $w_{\mu}^{\prime}(z) d z$ are preserved by conformal homeomorphisms, we can perform, if necessary, a fractional linear transformation to insure that both of the polynomials $p_{1}(z)$ and $p_{2}(z)$ are of the maximum possible degree $n-2$. The zeros of $p_{1}(z)$ and $p_{2}(z)$ will then be denoted respectively by $\xi_{1}, \xi_{2}, \cdots, \xi_{n-2}$, and $\zeta_{1}, \zeta_{2}, \cdots, \zeta_{n-2}$

We now assume that the zeros of $p_{1}(z)$ and $p_{2}(z)$ are distinct, and let $p(z)$ be a a polynomial with real coefficients of degree at most $2 n-4$. Observe that, for appropriately chosen constants $c, d_{j}$ and $e_{j}$, we can write 


$$
p(z)=p_{1}(z) p_{2}(z)\left[c+\sum_{j=1}^{n-2} \frac{d_{\perp}}{z-\xi_{j}}+\sum_{k=1}^{n-2} \frac{e_{\perp}}{z-\zeta_{k}}\right]
$$

But we then have the representation

$$
p(z)=c p_{1}(z) p_{2}(z)+p_{1}(z) r(z)+p_{2}(z) s(z)
$$

where $r(z)$ and $s(z)$ are certain polynomials of degree at most $n-$ 3 . Since the $p_{\mu}(z)$ are linearly independent, we can find real coefficients $a_{1}, \cdots, a_{n-1}$ and $b_{1}, \cdots, b_{n-1}$ such that

$$
\frac{p(z)}{q(z)}=c w_{1}^{\prime}(z) w_{2}^{\prime}(z)+w_{1}^{\prime}(z)\left(\sum_{j=1}^{n-1} a_{j} w_{j}^{\prime}(z)\right)+w_{2}^{\prime}(z)\left(\sum_{k=1}^{n-1} b_{k} w_{k}^{\prime}(z)\right),
$$

and so the functions listed in (8) do indeed span the linear space $\Gamma^{2}(D)$ and hence are linearly independent.

Lemma 2. If $D$ is a domain in the class $\Sigma_{n}$, and if $a<0$ and $b>0$, then for $w_{\mu}^{\prime}(z) d z$ and $w_{\nu}^{\prime}(z) d z$ distinct, the zeros of the differential $a w_{\mu}^{\prime}(z) d z+b w_{\nu}^{\prime}(z) d z$ are all real and simple.

Proof. Observe that the lemma is invariant under fractional linear transformations with real coefficients, so that we can assume, without loss of generality, that the point at infinity is in $D$. The boundary of $D$ is

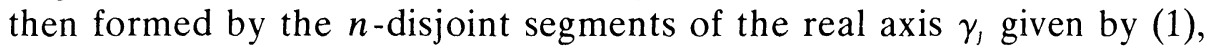
and we shall find it convenient to order the $\gamma_{j}$ by the condition (2). We have also the complementary segments $\alpha$, as in (3). By performing, if necessary, a second fractional linear transformation (with pole in $\alpha_{n-1}$ ), we can further assume that

$$
a w_{\mu}^{\prime}(z)+b w_{\nu}^{\prime}(z)=\frac{p(z)}{\sqrt{q(z)}},
$$

where $p(z)$ is a polynomial of the maximum possible degree, $n-2$. Let $N$ be the number of distinct real zeros of $a w_{\mu}^{\prime}(z)+b w_{\nu}^{\prime}(z)$. It is immediate that $n-2 \geqq N$, and it suffices to show that $N \geqq n-2$.

Consider the function

$$
\psi(z)=a \omega_{\mu}(z)+b \omega_{\nu}(z)
$$

$\psi(z)$ is harmonic in $D$ and continuous in the entire complex plane. If $\Psi(z)$ is the analytic completion of $\psi(z)$, then

$$
\Psi^{\prime}(z)=2 \frac{\partial}{\partial z} \psi(z)=\left(\frac{\partial}{\partial x}-i \frac{\partial}{\partial y}\right) \psi(z)=a w_{\mu}^{\prime}(z)+b w_{\nu}^{\prime}(z) .
$$


Moreover, since $\psi(z)=\psi(\bar{z})$, for $z=x$ taken from the real axis in $D$, it follows that

$$
\Psi^{\prime}(x)=\frac{\partial}{\partial x} \psi(x)
$$

Now let $m(\tau)$ be the number of endpoints of the interval $\alpha_{\tau}$ at which $\psi(z)$ is zero, and let $l(\tau)$ be the number of distinct solutions of $\psi(z)=0$ on the closed interval $\alpha_{\tau}$. Clearly, $l(\tau) \geqq m(\tau)$, and by virtue of equation (11), $\Psi^{\prime}(x)$ has at least $(l(\tau)-1)^{+}$distinct zeros on the interior of the interval $\alpha_{\tau}$.

Consider now the set

$$
\sigma=\{z \in D: \psi(z)=0\}
$$

and its closure $\bar{\sigma}$ in the extended complex plane $\hat{\mathbf{C}}$. Since on every analytic curve in $D$ which joins $\gamma_{\mu}$ to $\gamma_{\nu}$, we have a point $z$ at which $\psi(z)=0$, we see that $\bar{\sigma}$ is nonempty and indeed separates $\gamma_{\mu}$ from $\gamma_{\nu}$. In particular, $\bar{\sigma}$ contains a point on each of the connected components of $\left\{x\right.$ on the extended real line: $x \notin \gamma_{\mu}$ and $\left.x \notin \gamma_{\nu}\right\}$; call these points $x_{0}$ and $x_{1}$.

Next observe that on the set

$$
\hat{\sigma}=\{z \in \hat{\mathbf{C}}: \psi(z)=0\}=\sigma \cup\left(\bigcup_{j \neq \mu, \nu} \gamma_{j}\right)
$$

we have $\left(p^{2}(z) / q(z)\right) d z^{2} \leqq 0$. Thus $\hat{\sigma}$ is a system of orthogonal trajectories of the quadratic differential $\left(p^{2}(z) / q(z)\right) d z^{2}$. Suppose that $x_{0}$ is located on some $\gamma_{j}$. Then $x_{0}$ is the intersection of two distinct arcs of the orthogonal trajectory of $\left(p^{2}(z) / q(z)\right) d z^{2}$ and so $p\left(x_{0}\right)=0$, that is $\Psi^{\prime}\left(x_{0}\right)=$ 0 . The argument is similar for $x_{1}$. (For a discussion of the trajectories of quadratic differentials, see, for example, Jenkins [5].)

Case I. Suppose that the intervals $\gamma_{\mu}$ and $\gamma_{\nu}$ are adjacent, i.e., $\mu=\nu+1$. Then

$$
\begin{aligned}
& m(0)=m(1)=\cdots=m(\mu-2)=m(\mu+2)=\cdots=m(n-1)=2 \\
& m(\mu-1)=m(\mu+1)=1, \quad \text { and } \quad m(\mu)=0 .
\end{aligned}
$$

Certainly one of the points $x_{0}$ or $x_{1}$ is located on the interior of $\alpha_{\mu}$, say it is $x_{0}$. Then $l(\mu) \geqq 1$, and we consider separately the following two configurations:

(a) If $x_{1}$ is located on the interior of some segment $\alpha_{r}$, then $l(\tau) \geqq 1+m(\tau)$, and we have 
$n-2 \geqq N \geqq\left(\right.$ number of distinct zeros of $\Psi^{\prime}(z)$ on $\operatorname{int}\left(\alpha_{0} \cup \cdots \cup \alpha_{n-1}\right)$ )

$$
\begin{gathered}
\geqq \sum_{\rho=0}^{n-1} l(\rho)-n \geqq \sum_{\rho=0}^{n-1} m(\rho)+2-n=2(n-3)+2+2-n \\
=n-2,
\end{gathered}
$$

and $N=n-2$, as claimed.

(b) If $x_{1}$ is located on some closed segment $\bar{\gamma}_{r}$, then

$$
\begin{aligned}
& n-2 \geqq N \geqq 1+\left(\text { number of distinct zeros of } \Psi^{\prime}(z)\right. \text { on } \\
&\left.\quad \operatorname{int}\left(\alpha_{0} \cup \cdots \cup \alpha_{n-1}\right)\right) \\
& \geqq 1+\sum_{\rho=0}^{n-1} l(\rho)-n \geqq 1+\sum_{\rho=0}^{n-1} m(\rho)+1-n \\
&=1+2(n-3)+2+1-n=n-2,
\end{aligned}
$$

and so again $N=n-2$.

Case II. If the segments $\gamma_{\mu}$ and $\gamma_{\nu}$ are not adjacent, and if $\mu<\nu$, we then have

$$
\begin{aligned}
m(0)=m(1)=\cdots=m(\mu-2) & =m(\mu+1)=\cdots=m(\nu-2) \\
& =m(\nu+1)=\cdots=m(n-1)=2
\end{aligned}
$$

and

$$
m(\mu-1)=m(\mu)=m(\nu-1)=m(\nu)=1 .
$$

There are three possible configurations:

(a) If both $x_{0}$ and $x_{1}$ are on the interior of the set $\cup_{\rho=0}^{n-1} \alpha_{\rho}$, then $n-2 \geqq N \geqq$ (number of distinct zeros of $\Psi^{\prime}(z)$ on $\operatorname{int}\left(\alpha_{0} \cup \cdots \cup \alpha_{n-1}\right)$ )

$$
\begin{aligned}
& \geqq \sum_{\rho=0}^{n-1} l(\rho)-n \geqq \sum_{\rho=0}^{n-1} m(\rho)+2-n \\
& =2(n-4)+4+2-n=n-2,
\end{aligned}
$$

and we conclude that $N=n-2$.

(b) If one of the points $x_{0}$ or $x_{1}$ is located on the boundary of $D$, $\bigcup_{\rho=0}^{n-1} \gamma_{\rho}$, while the other point is located on interior of $\bigcup_{\rho=0}^{n-1} \alpha_{\rho}$, then

$$
\begin{aligned}
n-2 \geqq N+1+ & \text { (number of distinct zeros of } \Psi^{\prime}(z) \text { on } \\
& \left.\operatorname{int}\left(\alpha_{0} \cup \cdots \cup \alpha_{n-1}\right)\right)
\end{aligned}
$$




$$
\begin{aligned}
& \geqq 1+\sum_{\rho=0}^{n-1} l(\rho)-n \geqq 1+\sum_{\rho=0}^{n-1} m(\rho)+1-n \\
& =1+(2(n-4)+4)+1-n=n-2,
\end{aligned}
$$

so again $N=n-2$.

(c) Finally, if both $x_{0}$ and $x_{1}$ are located on the boundary of $D$, $\bigcup_{\rho=0}^{n-1} \gamma_{\rho}$, we have

$$
\begin{aligned}
& n-2 \geqq N \geqq 2+\left(\text { number of distinct zeros of } \Psi^{\prime}(z)\right. \text { on } \\
&\left.\quad \operatorname{int}\left(\alpha_{0} \cup \cdots \cup \alpha_{n-1}\right)\right) \\
& \geqq 2+\sum_{\rho=0}^{n-1} l(\rho)-n \geqq 2+\sum_{\rho=0}^{n-1} m(\rho)-n \\
&=2+(2(n-4)+4)-n=n-2,
\end{aligned}
$$

so again $N=n-2$.

All possible cases have now been treated so Lemma 2 is established.

Theorem 3. For $D$ a domain in the class $H_{n}$, no two distinct differentials $w_{\mu}^{\prime}(z) d z$ and $w_{\nu}^{\prime}(z) d z$ can possess a common zero.

Proof. Without loss of generality we can suppose that $D$ is in the class $\Sigma_{n}$, and suppose then that a point $z_{0}$ exists such that $w_{\mu}^{\prime}\left(z_{0}\right)=$ $w_{\nu}^{\prime}\left(z_{0}\right)=0$. From Corollary 1 we have $w_{\mu}^{\prime \prime}\left(z_{0}\right)<0$ and $w_{\nu}^{\prime \prime}\left(z_{0}\right)<0$, and we consider the differential

$$
\Psi^{\prime}(z) d z=\frac{w_{\mu}^{\prime}(z)}{w_{\mu}^{\prime \prime}\left(z_{0}\right)}-\frac{w_{\nu}^{\prime}(z)}{w_{\nu}^{\prime \prime}\left(z_{0}\right)} d z
$$

From Lemma 2 above $\Psi^{\prime}(z)$ has only simple zeros. However,

$$
\Psi^{\prime}\left(z_{0}\right)=\Psi^{\prime \prime}\left(z_{0}\right)=0
$$

and no such point $z_{0}$ can exist.

The following substitute for Noether's Theorem is now immediate.

THEOREM 4. If $D$ is a domain in the class $H_{n}, n \geqq 3$, then for an arbitrary enumeration of the boundary components of $D$, the quadratic differentials

$$
\begin{array}{r}
w_{1}^{\prime}(z)^{2} d z^{2}, w_{1}^{\prime}(z) w_{2}^{\prime}(z) d z^{2}, \cdots, w_{1}^{\prime}(z) w_{n-1}^{\prime}(z) d z^{2}, \\
w_{2}^{\prime}(z)^{2} d z^{2}, \cdots, w_{1}^{\prime}(z) w_{n-1}^{\prime}(z) d z^{2}
\end{array}
$$


are linearly independent and so span the space $\Gamma^{2}(D)$. If $n=2$, then $\Gamma^{2}(D)$ is spanned by the single quadratic differential $w_{1}^{\prime}(z)^{2} d z^{2}$, and if $n=1$, then $\Gamma^{2}(D)$ is vacuous.

Proof. The treatment of the cases $n=1$ and 2 is clear. For $n \geqq 3$ we can assume that $D$ is in the class $\Sigma_{n}$, and in this case the result is an immediate consequence of Lemma 1 and Theorem 3 .

The significance of Theorem 4 lies in the fact that the differentials $w_{\mu}^{\prime}(z), \mu=1,2, \cdots, n-1$, are up to a constant factor, the normal differentials of the first kind (with respect to an appropriately chosen canonical homology basis) on the Riemann surface obtained by forming the double of a domain $D \in H_{n}$. Theorem 4 tells us not only that we can choose a basis for $\Gamma^{2}(D)$ of the form (12), but also that we can do it arbitrarily and globally for all domains in $H_{n}$. Compare, for example, Bers [3].

Acknowledgment. I would like to thank the referee for his extremely helpful comments and criticisms concerning this paper.

\section{REFERENCES}

1. W. H. Barker, Plane domains with hyperelliptic double, Dissertation, Stanford University, 1975.

2. - Kernel functions in domain with hyperelliptic double, to appear.

3. L. Bers, Holomorphic differentials as functions of moduli, Bull. Amer. Math. Soc., 67 (1961), 206-210.

4. K. Hensel and G. Landsberg, Theorie der Algebraischen Funktionen einer Variablen, Leipzig, 1902.

5. J. Jenkins, Univalent Functions and Conformal Mappings, Springer-Verlag, Berlin, 1965.

6. G. Springer, Introduction to Riemann Surfaces, Addison-Wesley, Reading, Mass., 1957.

Received August 10, 1976. This work forms part of the author's Doctoral Dissertation at Stanford University written under the direction of M. M. Schiffer.

Daniel H. Wagner, Associates

Station Souare One

PAOLI, PA 19301 




\section{Pacific Journal of Mathematics \\ Vol. 70, No. $1 \quad$ September, 1977}

William H. Barker, Noether's theorem for plane domains with hyperelliptic

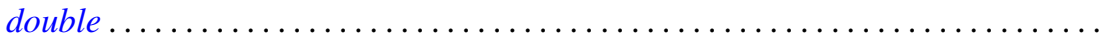

Michael James Beeson, Non-continuous dependence of surfaces of least area on the boundary curve ...................................... 11

Horst Behncke, Functions acting in weighted Orlicz algebras . . . . . . . . . . . . 19

Howard Edwin Bell, A commutativity study for periodic rings . . . . . . . . . . . 29

Peter Botta and Stephen J. Pierce, The preservers of any orthogonal group ....... 37

Douglas S. Bridges, The constructive Radon-Nikodým theorem ............. 51

James Dennis Brom, The theory of almost periodic functions in constructive

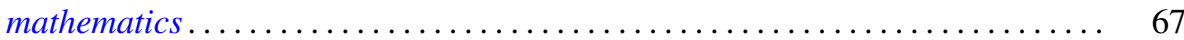

N. Burgoyne and C. Williamson, Semi-simple classes in Chevalley type groups ....

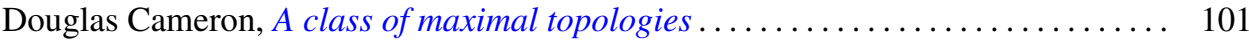

L. Carlitz, Enumeration of doubly up-down permutations . . . . . . . . . . . . . . 105

Paul Robert Chernoff, The quantum n-body problem and a theorem of

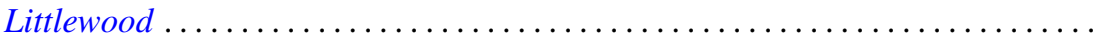

Jo-Ann Deborah Cohen, Locally bounded topologies on $F(X) \ldots \ldots \ldots \ldots \ldots \ldots$

Heinz Otto Cordes and Robert Colman McOwen, Remarks on singular elliptic

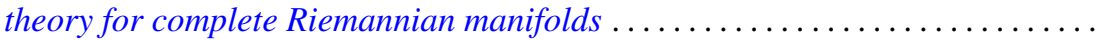

Micheal Neal Dyer, Correction to: "Rational homology and Whitehead

products"

Robert Fernholz, Factorization of Radonifying transformations

Lawrence Arthur Fialkow, A note on quasisimilarity. II ...... . .

Harvey Charles Greenwald, Lipschitz spaces of distributions on the surface of unit

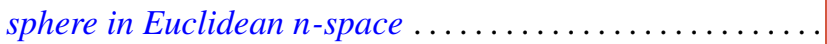

Albrecht Irle, On the measurability of conditional expectations

Tom (Roy Thomas Jr.) Jacob, Matrix transformations involving simple sequence

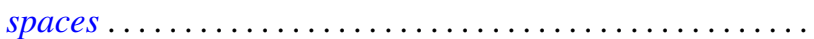

A. Katsaras, Continuous linear maps positive on increasing continuous

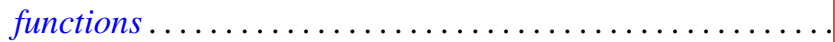

Kenneth Kunen and Judith Roitman, Attaining the spread at cardinals of cofinality

Lawrence Louis Larmore and Robert David Rigdon, Enumerating normal bundles

of immersions and embeddings of projective spaces ...... . .

Ch. G. Philos and V. A. Staïkos, Asymptotic properties of nonoscillatory solutions of differential equations with deviating argument .

Peter Michael Rosenthal and Ahmed Ramzy Sourour, On operator algebras containing cyclic Boolean algebras...

Polychronis Strantzalos, Strikt fast gleichgradig-stetige und eigentliche

Aktionen ...

Glenn Francis Webb, Exponential representation of solutions to an abstract

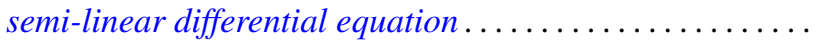

